\title{
On the Theory of Rational Insurance Purchasing in a Continuous-Time Model
}

\author{
by Eric Briys*
}

\section{Introduction}

The determination of the optimal insurance coverage has raised a growing academic interest. The seminal works by Arrow [1963], Smith [1968] and Mossin [1968] contributed to establish the cornerstone results of insurance economics. Indeed, it is now a well-known result that a risk averse expected utility maximizer will never purchase full insurance coverage if the insurance premium is actuarially unfair ${ }^{1}$. A positive amount deductible is always optimal from the insured's point of view if the premium is based on expected insurance payments and includes a positive proportional loading. Arrow [1974] extended this result to the case of state-dependent utility functions.

Some authors have however provided results which stand in sharp contrast with the above mentiond rule. Razin [1976], for instance, argued that the nature of results obtained very much depends on the decision framework. Using a binominal distribution of losses and Savage's minimax regret criterion as an alternative to the expected utility framework, he showed that it is always optimal to purchase insurance with a positive amount deductible even if the insurance premium is fair. In the same vein, Briys and Loubergé [1985] obtained that, under the Hurwicz criterion, full insurance is very often optimal even under a non zero insurance loading.

These results may seem conflicting. They nevertheless have a common basis: they focus on insurance in isolation. The optimal insurance coverage is determined in a single risk setting where consumption and portfolio decicions are not considered. In other words, previous studies assume either explicitly or implicitly that the insurance decision is perfectly separable from any other decisions. This strong assumption is obviously questionable. As recently pointed out by Doherty and Schlesinger [1983], the result by Smith and Mossin only holds if insurable and non insurable losses are non positively correlated. For a positive correlation between these losses more than full coverage is requested by risk averse individuals operating in incomplete markets.

* Assistant Professor of Finance, Centre HEC-ISA, France. The paper was presented at the 1986 Seminar of the European Group of Risk and Insurance Economists, in Hamburg (Germany), and at the 1987 Risk Theory Seminar, in Austin (Texas). Helpful comments from H. Loubergé, University of Geneva, H. Schlesinger, University of Alabama, R. Schöbel, University of Berlin, B. Solnik, Centre HEC-ISA, and D. Talay, INRIA are gratefully acknowledged. The original version of this research will be published in french in "L'Actualité Economique", Montreal, Canada. The usual caveat applies.

${ }^{1}$ An insurance premium is said to be actuarially unfair when it is based on expected insurance payments and includes a positive loading to cover for instance the administrative expenses of the company. 
Schulenburg [1986] proves similar results by noting that compulsory insurance has a non negligible impact on the free markets for insurance. Other scholars have also insisted on the necessity to include the insurance coverage decision into a portfolio framework. Mayers and Smith [1983] recognize that insurance is a special case of the risk hedging activity of the individual and suggest that other assets might be equally effective and perhaps less costly to fulfill the same task. Using a very similar framework Doherty [1984] has recently given an insightful analysis of the portfolio and insurance interdependencies.

But quite surprisingly no paper (except that of Richard [1975], which deals with life insurance under uncertain lifetime) explicitly accounts for the three dimensions of individual decision making in a continuous-time setting: consumption, portfolio and insurance. As early as 1969 , Gould argued that such an integrated approach would give the problem of the optimal insurance coverage a more realistic content. Dionne and Eeckhoudt [1984] suggested a very similar extension to their own results.

The purpose of the present paper is to develop such an extension and more specifically to derive optimum consumption, portfolio and damage insurance rules under continuoustime. Some references will be made to current practices in the homeowners insurance line as it provides a better understanding of our results.

The paper is organized as follows. The model and its main assumptions are presented in section 2. Our individual maximizes the expected utility of his consumption over his lifetime and trading in marketable assets takes place continuously. In section 3, optimal consumption, portfolio and insurance rules are derived and analyzed. The separability of the damage insurance demand is examined. Sections 4 and 5 present closed-form solutions under the isoelastic class of utility functions. In section 6 , a numerical illustration of the optimal insurance purchasing behavior is provided. The conclusion summarizes the main results and suggests further avenues of research.

\section{The model and the assumptions}

Our assumptions are close to those usually met in the financial literature. To simplify the analysis, we restrict the opportunity set of the individual to three assets. Two of them are risky while the third one is riskless. Both risky assets are assumed to be subject to "normal" sources of uncertainty described by a Wiener process plus a "damage" source of uncertainty described by a Poisson process.

The first asset is a marketable risky asset which is insurable. Indeed, this asset is subject to a potential damage. The value at time $t$ of this asset will be denoted by $S_{1}(t)$. We assume that $S_{1}(t)$ is generated by a so-called jump-diffusion process:

$$
d S_{1}(t)=\mu_{1} S_{1}(t) d t+\sigma_{1} S_{1}(t) d z_{1}(t)-S_{1}(t) d q(t)
$$

where $\mu_{1}$ and $\sigma_{I}^{2}$ are respectively the instantaneous expected percentage change in value of the first asset and its instantaneous variance conditional on no jump. $z_{1}(t)$ denotes a standard Wiener process. $q(t)$ is a Poisson process with parameter $\lambda$. Because a loss might be interpreted as a negative jump in the individual's wealth, the Poisson process is well adapted to our purpose. For the sake of simplicity we consider only two states of the world: full damage or no damage: the amplitude of the jump is one with probability one. 
The second asset is also risky and marketable. It is also subject to a risk of damage. We assume that its value $S_{2}(t)$ is generated by a mixed process:

$$
d S_{2}(t)=\mu_{2} S_{2}(t) d t+\sigma_{2} S_{2}(t) d z_{2}(t)-S_{2}(t) d q(t
$$

where $\mu_{2}, \sigma_{2}^{2}$ and $z_{2}(t)$ have the same meaning as in (1). The two Wiener processes $z_{1}(t)$ and $z_{2}(t)$ satisfy:

$$
\text { covariance }\left(d z_{1}, d z_{2}\right)=\varrho d t
$$

where $\varrho$ is the instantaneous correlation coefficient between $d z_{1}$ and $d z_{2}$. Note that the same Poisson process $q(t)$ with the same parameter is supposed to affect the second asset. This simplification has some intuitive appeal. There are many situations where a unique source of damage entails the simultaneous loss of many assets: fire, storm, theft, etc... Note also that by assumption the Wiener processes and the Poisson process are independent. In such a framework insurance will reveal useful since it is assumed to be the only available technology to hedge the jump dimension ${ }^{2}$ (see Mayers and Smith [1983]).

The third asset is a riskless asset whose riskless return per unit time is denoted $r$ :

$$
d S_{3}(t)=r S_{3}(t) d t
$$

In this three asset economy insurance coverage is available. Indeed the investor has the opportunity to mitigate the impact of jumps on his portfolio by purchasing insurance coverage. The insurance premium he has to pay is computed as follows:

$$
p\left[\beta_{1}(t), \beta_{2}\right]=(1+\theta) \lambda\left[\beta_{1}(t) a_{1}(t)+\beta_{2} a_{2}(t)\right] W(t)
$$

with $\beta_{1}$ : coinsurance rate on the first asset per unit time,

$\beta_{2}$ : regulated coinsurance rate on the second asset,

$\theta$ : insurance loading $\theta \geq 0$

$a_{1}$ : percentage of total wealth invested in the first asset,

$a_{2}:$ percentage of total wealth invested in the second asset,

$W$ : investor's total wealth.

Expression (4) deserves some explanation. Indeed, we assume that the only coinsurance rate over which the individual has control is $\beta_{1}$. The coverage on asset 2 is imposed by the insurance company to the individual. This kind of contractual practice is very usual in the homeowner insurance line. Insurance companies very often limit their coverage on certain assets. In France for instance, jewelries, furs, gold, silver, securities, paintings, collectibles are covered only up to $25 \%-30 \%$ of their market value. If the individual wishes to extend his coverage he needs an endorsement to his contract. We neglect here this latter possibility. Finally, one can observe that the introduction of $\beta_{2}$ enables also to consider the case of a non insurable asset (i.e. $\beta_{2}=0$ ).

A unique loading factor covering the administrative expenses and the risk of the insurance company is taken into account. In a subsequent section we relax this assumption and introduce two loading factors.

\footnotetext{
${ }^{2}$ Note also that the continuous-time analysis allows some useful insights. As recently shown by Jarrow and Rosenfeld [1984], jump risk (i.e. Poisson process) has non trivial implications for asset pricing models. Indeed, they show that jump risk is not diversifiable although they consider perfectly marketable assets.
} 
Using expressions (1) to (4) one can write down the wealth constraint of the individual which is given by the following stochastic differential equation:

$$
\begin{aligned}
d W(t)= & \left\{\left[\mu_{1} a_{1}(t)+\mu_{2} a_{2}(t)+\left(1-a_{1}(t)-a_{2}(t)\right) r\right] W(t)\right. \\
& \left.-p\left[\beta_{1}(t), \beta_{2}\right]-c(t)\right\} d t \\
& +\sigma_{1} a_{1}(t) W(t) d z_{1}(t)+\sigma_{2} a_{2}(t) W(t) d z_{2}(t) \\
& +\left[\beta_{1}(t)-1\right] a_{1}(t) W(t) d q(t)+\left[\beta_{2}(t)-1\right] a_{2}(t) W(t) d q(t)
\end{aligned}
$$

where $c$ denotes the consumption per unit time.

The individual's objective function is:

$$
\begin{array}{ccc}
\operatorname{Max} & E_{o} & \\
c, a_{1}, a_{2}, \beta_{1} & o &
\end{array}
$$

where $E_{o}$ is the conditional expectation operator given $W(0)=W_{0}$ as known. The time additive separable utility function $u$ is assumed to be strictly concave in the consumption per unit time.

The investor's lifetime is denoted $T$ and is supposedly known with certainty. We assume that there is no bequest left after the individual's death. The maximization (6) is subjected to (5). To keep the model in a very tractable form the investment opportunity set is supposed to be constant through time.

\section{Optimum consumption, portfolio and insurance rules}

We derive now the investor's optimal paths of consumption, investment and insurance over time. The technique used ther (namely stochastic dynamic programming) exactly parallels that of Merton [1971].

$$
\text { Let } \quad J[W(t), t]=\underset{c, a_{1}, a_{2}, \beta_{1}}{\operatorname{Max}} E_{t}^{T} u[c(s), s] d s .
$$

$J[W(t), t]$ is the indirect utility of wealth.

Let us define:

$$
\begin{aligned}
\phi\left(c, a_{1}, a_{2}, \beta_{1}, W, t\right)= & J_{t}+u(c) \\
& +\left[a_{1}\left(\mu_{1}-r\right) W+a_{2}\left(\mu_{2}-r\right) \mathrm{W}+\mathrm{rW}-(1+\theta) \lambda\left(\beta_{1} a_{1}+\beta_{2} a_{2}\right) W-c\right] J_{w} \\
& +1 / 2 J_{w w} W^{2}\left[\sigma_{1}^{2} a_{1}^{2}+2 \sigma_{1} \sigma_{2} \varrho a_{1} a_{2}+\sigma_{2}^{2} a_{2}^{2}\right] \\
& +\lambda\left[J\left(W+\left(\beta_{1}-1\right) a_{1} W+\left(\beta_{2}-1\right) a_{2} W\right)-J(W)\right]
\end{aligned}
$$

where the subscripts denote partial derivatives. Note that the time indices have been dropped for the sake of brevity.

The optimal controls $c^{*}, a_{1}^{*}, a_{2}^{*}, \beta_{1}^{*}$ are given by the extended version of Merton's theorem 1 (see Dreyfus [1965], p. 225 and Kushner [1967], chap. IV): 
Theorem 1: under our set of assumptions, the optimal controls $c^{*}, a_{1}^{*}, a_{2}^{*}, \beta_{1}^{*}$ are such that:

$$
0=\phi\left(c^{*}, a_{1}^{*}, a_{2}^{*}, \beta_{1}^{*}, W, t\right)>\phi\left(c, a_{1}, a_{2}, \beta_{1}, W, t\right), t \in[0, T]
$$

Theorem 1 implies that:

$$
0=\underset{c, a_{1}, a_{2}, \beta_{1}}{\operatorname{Max}} \phi\left(c, a_{1}, a_{2}, \beta_{1}, W, t\right)
$$

The first order conditions are:

$$
\begin{aligned}
0=\phi_{c}\left(c^{*}, a_{1}^{*}, a_{2}^{*}, \beta_{1}^{*}\right)= & u^{\prime}\left(c^{*}\right)-J_{w} \\
0=\phi_{a_{1}}\left(c^{*}, a_{1}^{*}, a_{2}^{*}, \beta_{1}^{*}\right)= & {\left[\left(\mu_{1}-r\right) W-(1+\theta) \lambda \beta_{1}^{*} W\right] J_{w} } \\
& +\left(\beta_{1}^{*}-1\right) W \lambda J_{w}\left[W+\left(\beta_{1}^{*}-1\right) a_{1}^{*} W+\left(\beta_{2}-1\right) a_{2}^{*} W\right] \\
& +\left(\sigma_{1}^{2} a_{1}^{*}+\sigma_{1} \sigma_{2} \varrho a_{2}^{*}\right) W^{2} J_{w w} \\
0=\phi_{a_{2}}\left(c^{*}, a_{1}^{*}, a_{2}^{*}, \beta_{1}^{*}\right)= & \left\{\left(\mu_{2}-r\right) W \cdot(1+\theta) \lambda \beta_{2}^{*} W\right\} J_{w} \\
& +\left(\beta_{2}-1\right) W \lambda J_{w}\left[W+\left(\beta_{1}^{*}-1\right) a_{1}^{*} W+\left(\beta_{2}-1\right) a_{2}^{*} W\right] \\
& +\left(\sigma_{2}^{2} a_{2}^{*}+\sigma_{1} \sigma_{2} \varrho a_{1}^{*}\right) W^{2} J_{w w} \\
& -(1+\theta) \lambda W a_{1}^{*} J_{w} \\
& +a_{1}^{*} \lambda W J_{w}\left[W+\left(\beta_{1}^{*}-1\right) a_{1}^{*} W+\left(\beta_{2}-1\right) a_{2}^{*} W\right] .
\end{aligned}
$$

Equations (8) and (9) are portfolio equations which reflects the availability of insurance coverage.

Equation (10) enables significant insights into the optimal demand for insurance. In a Mossin-Smith world an actuarial insurance policy should induce full insurance coverage, i.e. $\beta_{1}^{*}=1$. Plugging $\theta=0$ into (10) yields:

$$
0=-J_{w}+J_{w}\left[W+\left(\beta_{1}^{*}-1\right) a_{1}^{*} W+\left(\beta_{2}-1\right) a_{2}^{*} W\right]
$$

which is obviously satisfied when:

$$
\beta_{1}^{*}=1-\frac{\left(\beta_{2}-1\right)}{a_{1}^{*}} a_{2}^{*} .
$$

From (12) one can observe that the portfolio decision has an obvious feedback effect on the optimal insurance coverage. For strictly positive values of $a_{2}^{*}$ and for $\beta_{2}$ less than one, the optimal insurance coverage is greater than one ${ }^{3}$.

${ }^{3}(12)$ implies full insurance coverage on both assets. Indeed, the payment after a loss $a_{1}^{*}+a_{2}^{*}$. This should not come as a surprise since our framework corresponds to the case of perfect positive correlation of losses also examined by Doherty and Schlesinger [1983] in a monoperiodic setting. 
The case examined by Doherty and Schlesinger [1983] is easily grasped. A non insurable asset means $\beta_{2}=0$.

If so (12) is transformed into (12'):

$$
\beta_{1}^{*}=1+\frac{a_{2}^{*}}{a_{1}^{*}} .
$$

Theorem 2: Under our set of assumptions, the insurance decision is never separable from the portfolio decision and the prevailing insurance regulation $\left(\beta_{2}<1\right)$.

The traditional proposition by Mossin-Smith is relegated to a particular case as already noted by Doherty-Schlesinger [1983]. The optimal amount of insurance to be purchased is closely linked to other risk exposures in the portfolio.

A narrower specification of the insured's preferences brings additional insights into the various demand functions, especially when the insurance premium is unfair.

\section{Explicit solutions for a special class of utility functions}

As shown by Merton [1969] the system of equations (7), (8), (9), (10) is quite difficult to solve in general. However, if the utility function is assumed to be of the form yielding constant relative risk aversion, the system can be solved explicitly. Following Merton [1969, 1971] let $u(c)=\quad$ where $\eta=1-\gamma$ is the Arrow-Pratt measure of relative risk aversion supposed to be constant. Note that this specification has a sound empirical basis (see MorinSuarez [1982]).

After some computations the following optimal strategy is obtained ${ }^{4}$ :

$$
\begin{aligned}
& a_{1}^{*}=\frac{\left(\mu_{1}-r\right)-\lambda(1+\theta)}{(1-\gamma) \sigma_{1}^{2}\left(1-\varrho^{2}\right)}-\varrho \frac{\left(\mu_{2}-r\right)-\lambda(1+\theta)}{(1-\gamma)\left(1-\varrho^{2}\right) \sigma_{1} \sigma_{2}} \\
& a_{2}^{*}=\frac{\left(\mu_{2}-r\right)-\lambda(1+\theta)}{(1-\gamma) \sigma_{2}^{2}}-\frac{\sigma_{1}}{\sigma_{2}} \varrho a_{1}^{*} \\
& \beta_{1}^{*}=1+\frac{(1+\theta)^{\frac{\gamma-1}{-1}}}{a_{1}^{*}}-\frac{\left(\beta_{2}-1\right) a_{2}{ }^{*}}{a_{1}^{*}} \\
& c^{*}(t)=\frac{K}{(\gamma-1)-e^{-K /(\gamma-1)(T-t)}(\gamma-1)} W(t)
\end{aligned}
$$

${ }^{4}$ Note that for the sake of simplicity we assume that the individual's rate of impatience is zero. 
where $K=\gamma\left[\left(\mu_{1}-r\right) a_{1}^{*}+\left(\mu_{2}-r\right) a_{2}^{*}+r-(1+\theta) \lambda\left(\beta_{1}^{*} a_{1}^{*}+\beta_{2} a_{2}^{*}\right)\right.$

$$
\begin{aligned}
& \left.+1 / 2(\gamma-1)\left(a_{1}^{* 2} \sigma_{1}^{2}+2 \sigma_{1} \sigma_{2} \varrho a_{1}^{*} a_{2}{ }^{*}+\sigma_{2}^{2} a_{2}^{* 2}\right)\right] \\
& +\lambda\left[\left(1+\left(\beta_{1}^{*}-1\right) a_{1}^{*}+\left(\beta_{2}-1\right) a_{2}^{*}\right)^{\gamma_{-}} 1\right] .
\end{aligned}
$$

A third theorem can be formulated:

Theorem 3: For isoelastic marginal utility, $u^{\prime}(c)=c^{\gamma-1}, \gamma<1$, a unilateral separation between consumption and investment-insurance decisions obtains. Indeed, (13), (14) and (15) are independent of the consumption decision while (16) depends upon $a_{1}^{*}, a_{2}^{*}$ and $\beta_{1}^{*}$.

This theorem is very analog to the ones derived by Merton [1969] and by Briys [1985].

One can also note that the portfolio decision and the insurance purchase are independent of wealth. This was predictable from our constant relative risk aversion assumption.

Expression (15) deserves some further comments. An actuarial insurance policy $(\theta=$ $0)$ modifies (15) into:

$$
\beta_{1}^{*}=1-\frac{a_{2}^{*}\left(\beta_{2}-1\right)}{a_{1}^{*}} .
$$

The explicit optimal insurance coverage is identical to the one in (12). Such a result is hardly surprising since for an actuarial insurance policy the utility parameter vanishes (see (15)). Hence the optimal insurance decision under fair insurance pricing does not depend on the specific utility chosen.

Secondly it is interesting to observe that $\beta_{1}^{*}$ might be greater than one even under unfair insurance pricing. Two effects are at work. A risk effect induces the individual to ask for more coverage. A price effect through $\theta$ entails the possible optimality of a partial coverage. The offsetting behavior of these two effects determines $\beta_{1}^{*}$. A numerical application in section 6 illustrates this point.

\section{Explicit solutions under multiple loading insurance pricing}

The preceding analysis has supposed that a single loading is computed by the company for the two risks. This assumption can easily be relaxed by introducing two different loading factors in the insurance pricing schema. Expression (4) is transformed into:

$$
P\left(\beta_{1}, \beta_{2}\right)=\lambda\left[\left(1+\theta_{1}\right) \beta_{1} a_{1}+\left(1+\theta_{2}\right) \beta_{2} a_{2}\right] W
$$

where $\theta_{1}$ and $\theta_{2}$ are respectively the insurance loading for asset 1 and asset 2 . Differences in the cost of settlement of claims on asset 1 and 2 can for instance justify this new computation. 
Under the same preference assumption $\left(u^{\prime}(c)=c^{\gamma-1}, \gamma<1\right)$ as before the following optimal strategy is obtained ${ }^{5}$ :

$$
\begin{aligned}
& a^{*}{ }_{1}=\frac{\left\{\mu_{1}-r\right)-\lambda\left(1+\theta_{1}\right)}{\{1-\gamma) \sigma_{1}^{2}\left(1-\varrho^{2}\right)}-\varrho \frac{\left(\mu_{2}-r\right)-\lambda\left(1+\theta_{1}\right)-\lambda \beta_{2}\left(\theta_{2}-\theta_{1}\right)}{(1-\gamma)\left(1-\varrho^{2}\right) \sigma_{1} \sigma_{2}} \\
& a_{2}^{*}=\frac{\left(\mu_{2}-r\right)-\lambda\left(1+\theta_{1}\right)-\lambda \beta_{2}\left(\theta_{2}-\theta_{1}\right)}{(1-\gamma) \sigma_{2}^{2}\left(1-\varrho^{2}\right)}-\frac{\sigma_{1}}{\sigma_{2}} \varrho a_{1}^{*} \\
& \beta_{1}^{*}=1+\frac{\frac{1}{\gamma-1}}{a_{1}^{*}} \cdot \frac{\left(\beta_{2}-1\right) a_{2}^{*}}{a_{1}^{*}} \\
& c^{*}(t)=\frac{K}{(\gamma-1)-e^{-K /(\gamma-1)(T-t)}(\gamma-1)} W(t)
\end{aligned}
$$

where $K$ is defined as before except for $\theta$. When the loading factors are equal the new optimal strategy obviously reduces to the previous one.

The interesting feature in (19) and (20) is that the optimal portfolio decision now depends on $\beta_{2}$. This was not the case in (13) and (14). Schulenburg [1986] shows that the regulated insurance markets heavily influence the insurance purchasing behavior on the free markets for insurance. By introducing here the portfolio decision we extend his conclusions to the optimal holding of risky assets. Indeed, the partial derivatives of $a_{1}^{*}$ and $a_{2}^{*}$ with respect to $\beta_{2}$ capture this impact:

$$
\begin{aligned}
& \frac{\delta a_{1}^{*}}{\delta \beta_{2}}=\varrho \frac{\lambda\left(\theta_{2}^{-}-\theta_{1}\right)}{(1-\gamma)\left(1-\varrho^{2}\right) \sigma_{1} \sigma_{2}} \\
& \frac{\delta a_{2}^{*}}{\delta \beta_{2}}=-\frac{\lambda\left(\theta_{2}^{-}-\theta_{1}\right)}{(1-\gamma) \sigma_{2}^{2}\left(1-\varrho^{2}\right)} .
\end{aligned}
$$

When $\theta_{2}=\theta_{1}$, the insurance regulation has no effect on the market for assets. When $\theta_{2}$ and $\theta_{1}$ are not equal the response of $a_{1}^{*}$ to a change in $\beta_{2}$ depends on the sign of the correlation coefficient $p$ and on the respective magnitude of the two factors. As far as the second asset is concerned things are simpler since the sign of (24) only depends on that of $\left(\theta_{2}-\theta_{1}\right)$. Finally, the case of a zero correlation coefficient implies that the insurance regula-

$$
\begin{aligned}
& { }^{5} \text { When } \varrho=0 \text { and } \lambda=0 \text { it obtains: } \\
& a_{i}^{*}=\frac{\left(\mu_{i}-r\right)}{(1-\gamma) \sigma_{i}^{2}} i=1,2
\end{aligned}
$$

which is Merton's [1969] result. 
tion only affects the second asset. The introduction of the new insurance pricing scheme is consequently far from being innocuous.

\section{Numerical illustration}

The purpose of this section is to assess by the means of a numerical application the changes of the optimal insurance coverage in response to modifications in some exogenous parameters. Two cases will be distinguished. The first one concentrates on the optimal insurance demand under the single loading insurance pricing assumption. The second one deals with insurance demand under multiple loading insurance pricing.

In this first numerical solution, we use the optimal strategy (13), (14), (15) to identify how the insurance coverage behavior varies in response to changes in the loading factor:

\begin{tabular}{|ccccc|}
\hline \multicolumn{4}{c|}{ Table 1 } \\
Data: & Optimal Insurance Coverage as a Function of $\gamma$ and $\theta$ \\
& $\mu_{1}=12 \%$ & $\sigma_{1}=.10$ & $\varrho=0$ & $\lambda=.01$ \\
& $\mu_{2}=14 \%$ & $\sigma_{2}=.15$ & $r=10 \%$ & $\beta_{2}=.07$ \\
& Weak Risk Averter & Logarithmic & Strong Risk Averter \\
& & & \\
$\theta=15 \%$ & $1-\gamma=.35$ & $1-\gamma=1$ & $1-\gamma=3$ \\
$\theta=50 \%$ & 1.18 & 1.29 & 0.91 \\
& & & \\
& & &
\end{tabular}

Two comments on this table are in order. First, the price effect of $\theta$ is obvious: for every risk averse individual the optimal insurance coverage decreases. The result is strengthened by the negative impact of $\theta$ on $a_{1}^{*}$ and $a_{2}^{*}$. The portfolio dependence of the insurance decision is also clearcut: the less risk averse the individual, the greater $a_{1}^{*}$ and $a_{2}^{*}$ and the larger $\beta_{i}^{*}$. As this simple table shows, the optimal insurance coverage is the outcome of numerous interactions between risk aversion, portfolio decisions and insurance pricing.

In the second table, the individual's readiness to spend on insurance is shown to be related in a non trivial way to the relative risk exposure defined as the ratio $a_{2}^{*} / a_{1}^{*}$. To do this the optimal strategy (19), (20), (21) is used. Two loading assumptions are distinguished as well as two restrictions on the second asset coverage. 
Table 2

Optimal Insurance Coverage as a Function of $\gamma, \theta$ and $\beta_{2}$

Data:

$$
\begin{array}{llll}
\mu_{1}=12 \% & \sigma_{1}=.10 \quad \varrho=0 & \lambda=.01 \\
\mu_{2}=14 \% & \sigma_{2}=.15 \quad r=10 \% &
\end{array}
$$

Weak Risk Averter Logarithmic Strong Risk Averter

$$
1-\gamma=.35 \quad 1-\gamma=1 \quad 1-\gamma=3
$$

$\beta_{2}=0.1$

$\begin{array}{llll}\theta_{1}=30 \% ; \theta_{2}=15 \% & 2.29 & 2.22 & 2.20 \\ \theta_{1}=60 \% ; \theta_{2}=30 \% & 2.79 & 2.49 & 2.37\end{array}$

$\beta_{2}=0.8$

$\theta_{1}=30 \% ; \theta_{2}=15 \%$

1.09

1.03

0.99

$\theta_{1}=60 \% ; \theta_{2}=30 \%$

0.93

0.65

0.48

An anticipated result emerges in that table. Under our numerical assumption it is clear that the higher $\beta_{2}$, the lower the optimal insurance coverage for every risk averse individual and for a similarly loaded premium.

A closer examination of table 2 yields a quite striking result. Indeed, when $\beta_{2}=0.1$, the optimal insurance coverage increases with the two loading factors for every risk averse individual. On the other hand, a $\beta_{2}$ closer to one entails a reverse change: the optimal insurance coverage decreases.

The answer to this rather puzzling fact lies in the change of the relative risk exposure of the individual defined as $a_{2}^{*} / a_{1}^{*}$. Indeed, if one takes the example of the logarithmic individual things become clearer. For this individual the relative risk exposure under $\beta_{2}=0.1$, $\theta_{1}=30 \%, \theta_{2}=15 \%$ is equal to: 1.71 . When $\theta_{1}=60 \%$ and $\theta_{2} 30 \%$, the same ratio is equal to 2,7 . Now, if one applies the same computation for the case where $\beta_{2}=0.8$, it obtains respectively 1,17 and 1,78 . The increase in the relative risk exposure is simple to account for: when the loadings increase, the optimal holding of the first asset goes down sharply while the optimal holding of the second asset decreases only slightly. As a result, under a restrictive insurance regulation $\left(\beta_{2}=0.1\right)$ the insured is affected by almost the whole adverse affect of this risk exposure deterioration and is induced to ask for more coverage although 
the price is higher. On the other hand, when $\beta_{2}$ is closer to one, the effect of the relative risk exposure is not very strong and is dominated by the price effect. The individual asks for less coverage. This result mitigates Kahane and Koll's assertion that the amount of insurance purchase decreases with the loading factor (see their proposition 3.5, [1985]). Indeed, to derive their proposition they build a model where they claim that the single risky asset held by the insured can also be considered as a portfolio of individual assets (see their footnote 1). Such an assumption amounts to say that the risky portfolio allocation does not influence the optimal insurance coverage. In a simple two risky assets model it is shown here that this is rarely the case.

This short numerical simulation indicates that the determinants of the optimal insurance coverage are quite complex. Portfolio decisions have a non trivial impact on the optimal insurance demand. It comes out that even in the rather restrictive case of perfectly positively correlated jumps the main properties of the insurance decision are not as salient as some recent contributions might suggest. This last statement is all the more true since the numerical simulation presented above has used simplified versions of the optimal individual strategies where it was assumed for instance that the instantaneous correlation coefficient $\varrho$ was equal to zero.

\section{Conclusion}

This model generalizes earlier contributions in the insurance field. It provides a simultaneous treatment of consumption, portfolio and insurance decisions in a continuous-time setting. The optimal insurance purchasing has been thoroughly examined and shown to be strongly related to the various risk exposures of the individual's portfolio. Sufficient conditions for a separability between insurance and consumption have been given. One can also note that this research brings some additional light into Merton's contributions. Indeed, the original continuous-time framework by Merton has been enlarged to incorporate insurance coverages and insurance regulation. This last constraint has been shown to entail non negligible consequences on the optimal insurance purchasing behavior. More specifically, it has been shown by a numerical illustration that the optimal insurance demand is a far more complex topic than suspected so far.

Further avenues of research are of course possible. We mention only a few of them. Stochastic investment opportunities could have been introduced by making the $\mu_{i}, \sigma_{i}$ and $r$ dependent of some state variables. The model can also be extended by explicit consideration of the fact that income does not only stem from assets but also from wages.

Finally, we could have specified intertemporal dependent preferences à la Ryder et Heal [1973] by introducing the individual past consumption into the utility function. According to Sundaresan [1985] such an improvement dramatically changes the optimal consumption and portfolio choices. No doubt that it would also impact on the optimal insurance purchasing. 


\section{REFERENCES}

ARROW, K.J. [1963]: "Uncertainty and the welfare economics of medical care", American Economic Review, 53 (December 1963), 941-973.

ARROW, K.J. [1974]: "Optimal insurance and generalized deductibles”, Scandinavian Actuarial Journal, 1974, 1-42.

BRIYS, E. [1986]: "Insurance and consumption: the continuous-time case", Journal of Risk and Insurance, 53 (December 1986), 718-723.

BRIYS, E. and LOUBERGÉ, H. [1985]: “On the theory of rational insurance purchasing”, Journal of Finance, 40 (June 1985), 577-581.

DIONNE, G. and EECKHOUDT, L. [1984]: "Insurance and saving: some further results", Insurance:Mathematics and Economics, 3 (1984), 101-110.

DOHERTY, N. [1984]: "Portfolio efficient insurance buying strategies", Journal of Risk and Insurance, 51 (June 1984), 205-224.

DOHERTY, N. and SCHLESINGER, H. [1983]: "Optimal insurance in incomplete markets", Journal of Political Economy, 91 (December 1983), 1045-1054.

DREYFUS, S.V. [1965]: Dynamic Programming and the Calculus of Variations, Academic Press, New York.

FAMA, E. and MILLER, M. [1972]: The Theory of Finance, Holt Rinehart et Winston, New York.

GOULD, J. P. [1969]: "The expected utility hypothesis and the selection of optimal deductibles for a given insurance policy", Journal of Business, 42 (April 1969), 143-151.

JARROW, R. and ROSENFELD, E. [1984]: "Jump risks and the intertemporal capital asset pricing model", Journal of Business, 57 (July 1984), 337-351.

KUSHNER, H. J. [1967]: Stochastic Stability and Control, Academic Press, New York.

MARKOWITZ, H. [1952]: “Portfolio selection”, Journal of Finance, 7 (March 1952), 77-91.

MAYERS, D. and SMITH, C. [1983]: "The interdependance of individual portfolio decisions and the demand for insurance", Journal of Political Economy, 91 (April 1983), 304-311.

MERTON, R. [1969]: "Lifetime portfolio selection under uncertainty: the continuous-time case", Review of Economics and Statistics, 50 (August 1969), 239-246.

MERTON, R. [1971]: "Optimum consumption and portfolio rules in a continuous-time model", Journal of Economic Theory, 3 (1971), 373-413.

MORIN, R. A. and SUAREZ, A. F. [1983]: "Risk aversion revisited", Journal of Finance, 38 (September 1983), 1201-1216.

MOSSIN, J. [1968]: “Aspects of rational insurance purchasing”, Journal of Political Economy, 76 (July-August 1968), 553-568.

RAZIN, A. [1976]: "Rational insurance purchasing”, Journal of Finance, 31 (March 1976), 133-137. 
RICHARD, S. [1975]: “Optimal consumption, portfolio and life insurance rules for an uncertain lived individual in a continuous-time model”, Journal of Financial Economics, 2 (1975), 187-203.

RYDER, H. and HEAL, G. [1973]: "Optimal growth with intertemporally dependent preferences", Review of Economic Studies.

SCHULENBURG, M. [1986]: "Optimal insurance purchasing in the presence of compulsory insurance and insurable risks", Geneva Papers on Risk and Insurance, 11 (January 1986), 5-16.

SMITH, V. [1968]: "Optimal insurance coverage", Journal of Political Economy, 76 (January February 1968), 68-77.

SUNDARESAN, S. [1985]: "Intertemporally dependent preferences in the theories of consumption, portfolio choice and equilibrium asset pricing", Working Paper, 1985, Columbia University. 